\title{
Nearly Degenerate Four-Wave Mixing in Distributed Feedback Semiconductor Lasers Operating Above Threshold
}

\author{
Antonio Mecozzi, Alessandro D’Ottavi, and Rongqing Hui
}

\begin{abstract}
Nearly degenerate four-wave mixing in distributed feedback semiconductor lasers above threshold is investigated theoretically and experimentally. The experimental results reveal an almost symmetric amplification of probe and conjugate fields versus frequency with respect to zero pump-probe detuning, in contrast with observations in traveling wave amplifiers. The result of the theory, based on the coupled mode formalism, is a set of nonlinear differential equations which are solved in the mean field approximation. The theory is shown to match well with the experimental results when the effect of the nonlinear gain compression is taken into account.
\end{abstract}

\section{INTRODUCTION}

$\mathrm{D}$ EGENERATE four-wave mixing (DFWM) and nearly degenerate four-wave mixing (NDFWM) have attracted considerable attention in nonlinear optics. DFWM has found several important applications in phase conjugation, extensively used in real-time holography and adaptive optics [1], [2]. In these applications amplifying media are preferred in order to realize high-efficiency mixing. In this framework, colinear intracavity four-wave mixing in Fabry-Perot (FP) semiconductor lasers above threshold has been experimentally reported [3]-[6]. In such lasers the feedback due to the cavity leads to a higher four-wave mixing efficiency and conjugate amplification up to $30-40 \mathrm{~dB}$ has been obtained.

The theoretical approach to explain these experimental results has been qualitatively outlined by Bogatov et al. [7] by treating the semiconductor laser as an inverted twolevel system. They put forward a calculation of the carrier rate equation coupled with nonlinear Maxwell equations to explain the interaction of two modes by the saturation induced index change. Recently, this theory has been extended to the analysis of NDFWM process in the traveling wave semiconductor amplifier (TWA) [8] and several investigations on NDFWM in TWA's with different pump configurations have been published later on [9]-[11].

This paper is devoted to give both an experimental and a theoretical analysis of NDFWM in a distributed feedback laser (DFB) where, like in FP lasers, the FWM ef-

Manuscript received May 1, 1992; revised November 13, 1992. This work was carried out in the framework of the agreement between the Fondazione Ugo Bordoni and the Italian Post and Telecommunication Administration.

The authors are with Fondazione Ugo Bordoni, 00142 Rome, Italy. IEEE Log Number 9209102. ficiency is enhanced by the cavity feedback mechanism. DFB lasers are particularly attractive since they oscillate in a single mode [12], which can act as a pump in FWM. In this configuration a probe signal slightly detuned with respect to the oscillating mode gives rise to a conjugate signal. The amplification of probe and conjugate waves in lasers above threshold is symmetric with respect to zero probe-pump detuning and the effective FWM bandwidth is mainly dependent on the relaxation oscillation frequency of the pump laser. These properties are different from those obtained in TWA's where the probe amplification is typically asymmetric [8]-[11] and the effective FWM bandwidth is only determined by the carrier's spontaneous lifetime [8].

The paper is divided in seven parts. In Section II we present the experimental results. In Section III we develop a detailed theory of nearly degenerate four-wave mixing in semiconductor lasers above threshold based on the coupled-mode formalism. The main result of the theory is a set of coupled nonlinear differential equations for the spatial evolution of pump, probe and conjugate fields in the cavity. The effects of the nonlinear gain compression and of the spatial hole burning are taken into account. These effects, although present also in FWM of TWA's, are particularly important in semiconductor lasers above threshold because without them the predicted enhancement of the FWM efficiency at the laser resonance frequency would be much larger than actually observed. In Section IV we obtain an explicit expression for the input-output fields for the case in which the fields inside the cavity can be assumed as uniform (homogeneous field approximation). In Section $V$ the effect of noise on the amplification of probe and conjugate fields is introduced. In Section VI we compare theory and experimental results. In Section VII we report our conclusions.

\section{EXPERIMENT}

The experimental setup is shown in Fig. 1. Two identical DFB-BH laser diodes with an emission wavelength of $1554 \mathrm{~nm}$ were used. Two diffraction limited lenses with a numerical aperture of $0.65 \mathrm{NA}$ were used for the beam coupling and two optical isolators, inserted between the laser generating the probe wave (probe laser) and the laser generating the FWM (test laser), provided more than 50 


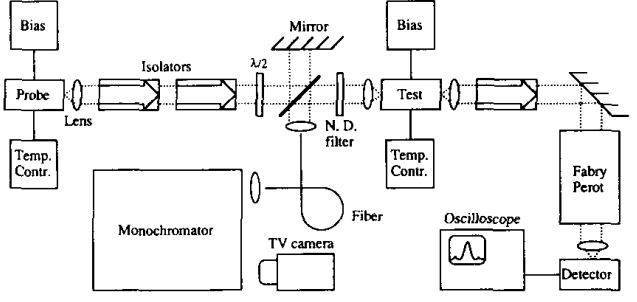

Fig. 1. Experimental setup.

$\mathrm{dB}$ of isolation. A half-wavelength plate was used to match the polarization of the two lasers. A variable density filter was inserted between probe and test lasers in order to adjust the intensity of the probe beam. The beams from the two lasers are mixed on a beam splitter and sent to a monochromator for a coarse adjustment of the emission wavelengths. Frequency matching and adjusting is accomplished by controlling the lasers' heat-sink temperature. The relative amplitude of the probe and conjugate beams was measured by means of a Fabry-Perot interferometer placed behind the rear facet of the test laser. The evaluation of the optical power actually injected into the test laser cavity is very difficult. The use of the method proposed by Mukai and Yamamoto [13] for evaluating the injected optical power in FP semiconductor lasers becomes very difficult for a DFB laser that works below threshold since the gain difference between the pass-band and the stop-band is too large. Initially, the coupled optical power was roughly estimated from the photocurrent induced in the test laser at zero bias. This method is quite easy but it is indeed only qualitative. A more accurate evaluation of the injected optical power was performed by using injection locking. This technique allows an accurate measurement of the linewidth enhancement factor $\alpha$ [14]. The knowledge of the $\alpha$ value allows to evaluate the injected optical wave power once the injection locking bandwidth is measured [15]. The probe optical injection was maintained to about $0.02 \mu \mathrm{W}$. In such a condition, the probe induced depletion in the test laser is very weak and can be neglected when the pump-probe detuning is large. However, when the detuning approaches the injection locking boundary, the pump depletion is still evident.

Another problem arises when the test laser is biased relatively close to threshold so that, for moderate detuning, injection locking and partial injection locking are easily established. In order to avoid these unwanted effects and focus the attention on FWM, the test laser was biased at relatively high levels. The experimental results obtained for two different values of the test-laser bias are shown in Fig. 2. It is worth to notice that the probe and conjugate wave amplification, $30 \mathrm{~dB}$ at least, is almost symmetrical with respect to the zero detuning. This result is different from that obtained in the TWA's where asymmetric probe amplification has been observed [8]-[11]. This almost-symmetrical amplification is due to the effect of the cavity, where strong coupling between pump,

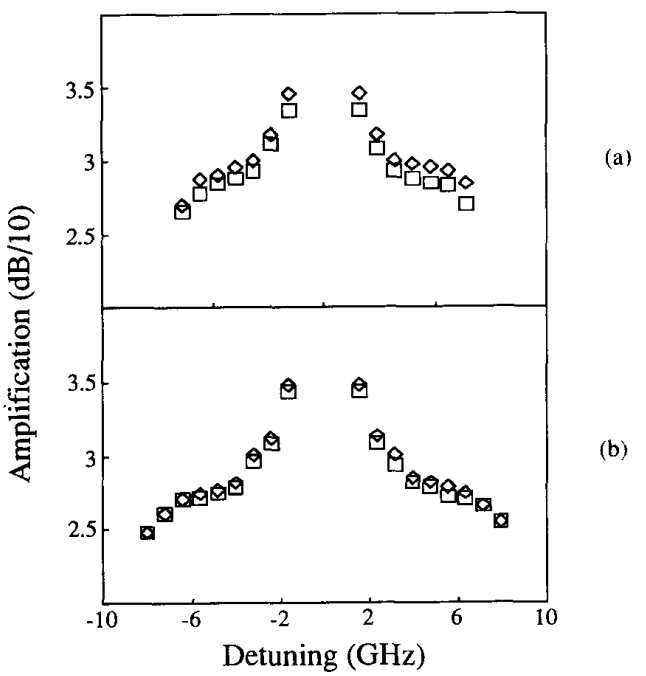

Fig. 2. Measured probe (diamonds) and conjugate (squares) wave amplifications with the injected probe optical power of $0.02 \mu \mathrm{W}$ and the test laser biased at (a) $I=50 \mathrm{~mA}$ and (b) $I=70 \mathrm{~mA}$. The test laser has the threshold current of $21 \mathrm{~mA}$.

probe, and conjugated wave occurs close to the resonance peak of the laser cavity.

When the test laser is biased at low current, the probe and the conjugate amplifications are different and the effective FWM bandwidth is relatively small as shown in Fig. 2(a). Increasing the bias current of the test laser, the difference in the amplification of probe and conjugate waves becomes less evident and the laser relaxation resonance moves at a higher frequency. This is illustrated in Fig. 2(b). As already shown in FP semiconductor lasers [4], [5], systematic measurements indicate that the effective FWM bandwidth in DFB semiconductor lasers working above threshold depends mainly on the test laser relaxation oscillation frequency, while in the TWA's, this effective FWM bandwidth is determined by the carrier's spontaneous lifetime as stated in several previous papers [8]-[11]. Because of the dynamic damping effect, side wings corresponding to the relaxation oscillation become less pronounced increasing the pump power. The width of the plateaus on the wings can be larger than $1 \mathrm{GHz}$ when the pump power is high.

\section{Coupled Wave Equations Analysis}

The theory of FWM has been already developed by Agrawal in [8]. Even though the analysis in that paper is general, most of the attention is devoted to the discussion of FWM in semiconductor laser amplifiers. In semiconductor lasers above threshold the FWM efficiency is modified by the cavity and a more detailed theory is required. The reason for this need is readily understood. It is the optical modulation of the carrier density that leads to the FWM interaction. It is well known that the response to a current modulation of a semiconductor laser above threshold is enhanced close to the relaxation oscillation fre- 
quency. The phase noise spectrum, which can be seen as the modulation response to the broad-band excitation due to the spontaneous emission, is also enhanced at the same frequency. An enhancement of the FWM efficiency at the resonance frequency is then expected, has been previously observed [4], [5], and is confirmed by our measurements. It has been already pointed out that if we assume, in a rate equation approach, that the laser gain is only a function of the carrier density the enhancement of the response to a current modulation is higher than that actually observed [16], [17]. The same has to be true for the enhancement of the FWM efficiency. Different mechanisms have been proposed so far to explain this discrepancy. One is based on a modification of the material gain of a semiconductor laser by introducing an explicit dependence on the laser intensity. The physical origin of this term can be either spectral hole burning [18], [19] or carrier heating [20], [21]. Another mechanism is based on the spatial hole burning induced by the cavity standing wave [22]. While these effects are expected to be important for semiconductor lasers above threshold, in semiconductor laser amplifiers they are of second order because their importance is restricted to a spectral region (tens of GHz) where the FWM efficiency of semiconductor amplifiers is negligible. Since we are interested in this paper to FWM in semiconductor lasers above threshold, we will introduce both these effects in our model.

In a semiconductor laser in which only the fundamental waveguide TE mode is present the evolution of the field $E$, which through the paper will be normalized such that $|E|^{2}$ is the power flux in units of $\mathrm{W} / \mathrm{m}^{2}$, is described by the wave equation

$$
\nabla^{2} E-\frac{n^{2}}{c^{2}} \frac{\partial^{2} E}{\partial t^{2}}=\frac{1}{\epsilon_{o} c^{2}} \frac{\partial^{2} P}{\partial t^{2}}
$$

where $n$ is the refractive index, $\epsilon_{o}$ is the vacuum permittivity, and $c$ is the velocity of light in vacuum. The total intracavity field is

$$
E=\frac{1}{\sqrt{\epsilon_{o}}} U(x, y) \sum_{j} E_{j}(z) e^{-i \omega_{j} t}
$$

where $U(x, y)$ is the transverse distribution of the TE mode. The subscript $j=0,1,2$ correspond to pump, probe, and conjugate wave, respectively, whose frequencies obey the relationship

$$
\omega_{1}-\omega_{0}=\omega_{o}-\omega_{2}=\Omega
$$

The matter polarization $P=\epsilon_{o} \chi E$ is expanded in the Fourier components at the same frequencies of the fields

$$
P=U(x, y) \eta(x, y) \sum_{j} P_{j}(z) e^{-i \omega_{j} t}
$$

$\eta(x, y)$ being a step function assuming the value 1 in the active region and 0 elsewhere, introduced to account for the field-matter interaction only in the region where it is enhanced by the presence of the resonant medium. Strictly speaking, this expression does not hold when the matter susceptibility $\chi$ is a function of the field, as in semiconductor lasers. We will discuss this issue later on.

In a DFB laser, the grating introduces a longitudinal perturbation of the refractive index of period $\Lambda$. If we keep only the first term in the Fourier expansion we have

$$
n(z)=n+\frac{\Delta n}{2} \cos \left(2 k_{D} z+\phi\right)
$$

being $k_{D}=\pi / \Lambda$ the Bragg wavenumber, $\phi$ is the grating phase, and $\Delta n$ is the amplitude of the modulation. We enter expansions (2) and (4) into the wave equation (1), the eliminate the $x-y$ dependence through multiplication by $U^{*}(x, y)$ and integration over the transverse coordinates. The result is

$\frac{d^{2} E_{j}}{d z^{2}}+k_{j}^{2} E_{j}+4 k_{j} \kappa \cos \left(2 k_{D} z+\phi\right) E_{j}=-\frac{\omega_{j}^{2} \Gamma}{\epsilon_{o} c^{2}} P_{j}$

where $k_{j}=\bar{n} \omega_{j} / c=\omega_{j} / v_{g}$ are the wavenumbers, $\bar{n}$ is the group refractive index pertaining to the waveguide mode, $v_{g}$ is the group velocity in the waveguide, $\kappa=\Delta n \omega /(4 c)$ is the coupling coefficient of the DFB grating and the waveguide confinement factor $\Gamma$ is defined as

$$
\begin{aligned}
\Gamma & =\frac{\int d x \int d y \eta(x, y)|U(x, y)|^{2}}{\int d x \int d y|U(x, y)|^{2}} \\
& =\frac{\int d x \int d y \eta(x, y)|U(x, y)|^{2}}{A_{\text {eff }}}
\end{aligned}
$$

$A_{\text {eff }}$ being the effective area of the mode.

In semiconductor lasers, the interaction field matter is realized through the carrier density $N$ which, in a rate equation model, obeys the equation

$$
\frac{d N}{d t}=\frac{I}{q V}-\frac{N}{\tau_{s}}-\frac{g}{\hbar \omega_{o}}|E|^{2}+D \nabla^{2} N
$$

where $I$ is the injection current, $q$ is the electron charge, $V$ is the active volume of the device, $\tau_{s}$ is the lifetime of the minority carriers, $g$ is the optical gain in $\mathrm{cm}^{-1}$, and $D$ is the diffusion coefficient.

We assume that the dependence of $g$ on the carrier density and on the field intensity is

$$
g=a\left(N-N_{o}\right)\left(1-\epsilon|E|^{2}\right)
$$

where $a$ is the gain coefficient, $N_{o}$ is the carrier density at transparency and $\epsilon$ is the gain compression factor. The actual dependence of the gain on the field intensity is still object of controversy. We have used here a linearized expression which restrict ourselves to the case $\epsilon|E|^{2}<<$ 1 .

We introduce expansion (2) into the rate equation (8). The time independent component of the electric field and the beating between pump and probe at the frequency difference $\Omega$ produce a time independent bias and a modu- 
lation at frequency $\Omega$ of the carrier density. Both components are, strictly speaking, function of the transverse coordinates $(x, y)$. The effect of diffusion is to average the carrier density over the transverse dimensions. If we assume that the transverse dimensions of the laser are smaller than the diffusion length (we are not considering here broad-area lasers) the dependence of the carrier density on $x$ and $y$ can be removed and we can write

$$
N=\bar{N}(z)+\left[\Delta N(z) e^{-i \Omega t}+\Delta N^{*}(z) e^{i \Omega t}\right] .
$$

The values of the time independent component of the carrier density $\vec{N}$ and of the modulation $\Delta N$ are found introducing expansion (2) and (10) into the rate equation (8) and integrating over the transverse dimensions. If we consider the probe and conjugate fields as perturbations of the total intracavity field, we obtain to lowest order

$$
\left(1+\left|E_{o}\right|^{2} / P_{s}\right) \bar{N}=I \tau_{s} /(q V)+N_{o}\left|E_{o}\right|^{2} / P_{s}+\tau_{s} D \nabla^{2} \bar{N}
$$

and to first order

$$
\begin{aligned}
\left(1+\left|E_{o}\right|^{2} / P_{s}-i \Omega \tau_{s}\right) \Delta N= & C\left(\bar{N}-N_{o}\right)\left(E_{o} E_{1}^{*}\right. \\
& \left.+E_{o}^{*} E_{2}\right) / P_{s}+\tau_{s} D \nabla^{2} \Delta N
\end{aligned}
$$

where $P_{s}$ is the saturation intensity

$$
P_{s}=\frac{\hbar \omega_{o}}{\Gamma a \tau_{s}} \text {. }
$$

The factor $C$ is introduced phenomenologically to account for the nonplanewave nature of the waveguide mode and for the consequent nonperfect overlapping of the fields at different frequencies. The waveguide confinement factor $\Gamma$ appears in (13) since we chose to refer the power flux $|E|^{2}$ to the effective area of the mode.

The spatial dependence of pump, probe and conjugate field is assumed to be

$$
E_{j}(z)=\sqrt{P_{s}}\left[A_{j}^{+}(z) e^{i k_{D} z}+A_{j}^{-}(z) e^{-i k_{D} z}\right] .
$$

The intensity of the component at each frequency is modulated with a beatlength which is half of the Bragg wavenumber, and so is the carrier density. Even though the carrier density grating so generated is smoothed by carrier diffusion, since it has a periodicity of the order of the diffusion length $\sqrt{D \tau_{s}}$, its effect cannot be neglected. As already mentioned, in fact, the effect of the carrier grating in semiconductor lasers above threshold is to damp the resonant enhancement of the FWM efficiency at the laser resonance frequency.

The effect of the standing waves generated by the counterpropagating fields on the carrier density is accounted for by expanding the coefficients of (10) as

$$
\begin{aligned}
\bar{N}(z) & =\bar{N}_{b}(z)+\bar{N}_{+}(z) e^{2 i k_{D} z}+\bar{N}_{-}(z) e^{-2 i k_{D} z} \\
\Delta \mathrm{N}(z) & =\Delta N_{b}(z)+\Delta N_{+}(z) e^{2 i k_{D} z}+\Delta N_{-}(z) e^{-2 i k_{D} z}
\end{aligned}
$$

and taking into account the only relevant effect of diffusion to smooth the carrier density gratings, by approximating

$$
\begin{aligned}
\nabla^{2} \bar{N}(z) & =-4 k_{D}^{2}\left[\bar{N}_{+}(z) e^{2 i k_{D} z}+\bar{N}_{-}(z) e^{-2 i k_{D} z}\right] \\
\nabla^{2} \Delta N(z) & =-4 k_{D}^{2}\left[\Delta N_{+}(z)^{2 i k_{D} z}+\Delta N_{-}(z) e^{-2 i k_{D} z}\right] .
\end{aligned}
$$

The effect of diffusion can be handled by a perturbative approach. The amplitude of the $z$ independent components is calculated in the limit of $D=\infty$ by inserting expansion (14) for the pump field into equations (11) and (12), and neglecting all the terms due to the beating of the counterpropagating waves. The result is

$$
\begin{aligned}
\bar{N}_{b} & =N_{o}+\frac{I \tau_{s} /(q V)-N_{o}}{1+P_{o}} \\
\Delta N_{b} & =\frac{C\left(\bar{N}_{b}-N_{o}\right)}{1+P_{o}-i \Omega \tau_{s}} S_{o}
\end{aligned}
$$

where

$$
\begin{aligned}
& P_{o}=\left|A_{o}^{+}\right|^{2}+\left|A_{o}^{-}\right|^{2} \\
& S_{o}=A_{o}^{+}\left(A_{1}^{+}\right)^{*}+A_{o}^{-}\left(A_{1}^{-}\right)^{*}+\left(A_{o}^{+}\right)^{*} A_{2}^{+}+\left(A_{o}^{-}\right)^{*} A_{2}^{-} .
\end{aligned}
$$

The coefficient of the spatial harmonic terms are then obtained by entering into (11) and (12) the full expressions (15) and (16) along with expansion (14) for the fields, and making use of the obtained results. Selecting in both sides of the equations the coefficients of the spatial harmonic functions with the same wavevectors, we find

$$
\begin{aligned}
\bar{N}_{+} & =-\xi Q_{o} \frac{\bar{N}_{b}-N_{o}}{1+\xi P_{o}}, \quad \bar{N}_{-}=\bar{N}_{+}^{*} \\
\Delta N_{+} & =\xi \frac{C\left(\bar{N}_{b}-N_{o}\right) S_{2}+\Delta N_{b} Q_{o}}{1+\xi\left(P_{o}-i \Omega \tau_{s}\right)} \\
\Delta N_{-} & =\xi \frac{C\left(\bar{N}_{b}-N_{o}\right) S_{1}+\Delta N_{b} Q_{o}^{*}}{1+\xi\left(P_{o}-i \Omega \tau_{s}\right)}
\end{aligned}
$$

where

$$
\begin{aligned}
\xi & =\frac{1}{1+4 D \tau_{s} k_{D}^{2}} \\
Q_{o} & =A_{o}^{+}\left(A_{o}^{-}\right)^{*} \\
S_{1} & =\left(A_{o}^{+}\right)^{*} A_{1}^{-}+A_{o}^{-}\left(A_{2}^{+}\right)^{*} \\
S_{2} & =\left(A_{o}^{-}\right)^{*} A_{1}^{-}+A_{o}^{+}\left(A_{2}^{-}\right)^{*} .
\end{aligned}
$$

Let us address now the interaction between the field and the active medium. The field-carrier interaction is governed by the relation

$$
P=\epsilon_{o} \chi E
$$

where the matter susceptibility $\chi=\chi_{r}+i \chi_{i}$ is expressed as

$$
\chi_{i}=-\frac{n c}{\omega_{o}} g=-\frac{n c}{\omega_{o}}\left(1-\epsilon|E|^{2}\right) a\left(N-N_{o}\right)
$$




$$
\chi_{r}=-\frac{n c}{\omega_{o}}\left(\alpha-\epsilon \beta|E|^{2}\right) a\left(N-N_{o}\right) .
$$

$\alpha$ being the Henry's linewidth enhancement factor and $\beta$ is the nonlinear linewidth enhancement factor. Introducing (2) and (10) into the definition of the polarization (28) we obtain a decomposition of $P$ in Fourier modes at the frequencies $\omega_{j}$. Without gain compression this decomposition has the form of (4), because the carrier density $N$ is uniform on the transverse dimensions of the active region so that $P$ depends on the transverse coordinates only through the linear dependence on the field $E$. If the gain compression factor $\epsilon$ is different from zero, (4) does not hold rigorously because the gain saturation term in the polarization has spatial dependence $|U(x, y)|^{2} U(x, y)$. Yet, the deviation from (4) can be handled perturbatively by substituting the actual gain compression factor with an effective value which depends on the waveguide mode.

Similarly to what already done for the field, we expand the Fourier amplitudes of the polarization in two counterpropagating components

$$
P_{j}=P_{j}^{+} e^{i k D z}+P_{j}^{-} e^{-i k D z} \text {. }
$$

The values of the $P_{j}^{+}$'s and $P_{j}^{-}$'s are found by entering expansions of the carrier density (10), (15), and (16) and expansion of the field (2) into the definition of the polarization (28), multiplying by $U^{*}(x, y)$ and integrating over the transverse dimensions. After some algebra we get

$$
\begin{aligned}
P_{o}^{+}= & \epsilon_{o} A P_{s}^{1 / 2}\left[a\left(\bar{N}_{b}-N_{o}\right) A_{o}^{+}+a \bar{N}_{+} A_{o}^{-}\right] \\
& -\epsilon \epsilon_{o} B P_{s}^{3 / 2} a\left(\bar{N}_{b}-N_{o}\right)\left(P_{o}+\left|A_{o}^{-}\right|^{2}\right) A_{o}^{+} \\
P_{1}^{+}= & \epsilon_{o} A P_{s}^{1 / 2}\left[a\left(\bar{N}_{b}-N_{o}\right) A_{1}^{+}+a \bar{N}_{+} A_{1}^{-}\right. \\
& \left.+a \Delta N_{b} A_{o}^{+}+a \Delta N_{+} A_{o}^{-}\right] \\
& -\epsilon \epsilon_{o} B P_{s}^{3 / 2}\left[2 P_{o} A_{1}^{+}+2 A_{o}^{+}\left(A_{o}^{-}\right)^{*} A_{1}^{-}\right. \\
+ & \left.2 A_{o}^{+} A_{o}^{-}\left(A_{2}^{-}\right)^{*}+\left(A_{o}^{+}\right)^{2}\left(A_{2}^{+}\right)^{*}\right] \\
P_{2}^{+}= & \epsilon_{o} A P_{s}^{1 / 2}\left[a\left(\bar{N}_{b}-N_{o}\right) A_{2}^{+}+a \bar{N}_{+} A_{2}^{-}\right. \\
& \left.+a \Delta N_{b} A_{o}^{+}+a \Delta N_{+} A_{o}^{-}\right] \\
& -\epsilon \epsilon_{o} B P_{s}^{3 / 2}\left[2 P_{o} A_{2}^{+}+2 A_{o}^{+}\left(A_{o}^{-}\right)^{*} A_{2}^{-}\right. \\
& \left.+2 A_{o}^{+} A_{o}^{-}\left(A_{1}^{-}\right)^{*}+\left(A_{o}^{+}\right)^{2}\left(A_{1}^{+}\right)^{*}\right]
\end{aligned}
$$

where

$$
\begin{aligned}
& A=-\frac{n c}{\omega_{o}}(\alpha+i) \\
& B=-\frac{n c}{\omega_{o}}(\beta+i)
\end{aligned}
$$

and the effective gain compression factor

$$
\epsilon \rightarrow \epsilon \frac{\int d x \int d y \eta(x, y)|U(x, y)|^{4}}{\int d x \int d y \eta(x, y)|U(x, y)|^{2}}
$$

substitutes the original value. The corresponding polarizations labeled with - are found by the permutation ($\leftrightarrow+$ ).
Introducing expansions (14) and (31) in (6) and applying the slowly varying amplitude and the rotating wave approximations we get

$$
\begin{aligned}
\frac{d A_{j}^{+}}{d z}-i \Delta k_{j} A_{j}^{+}-i \kappa A_{j}^{-} & =-i \frac{\omega_{j} \Gamma}{2 \epsilon_{o} c \bar{n} \sqrt{P_{s}}} P_{j}^{+} \\
-\frac{d A_{j}^{-}}{d z}-i \Delta k_{j} A_{j}^{-}-i \kappa A_{j}^{+} & =-i \frac{\omega_{j} \Gamma}{2 \epsilon_{o} c \bar{n} \sqrt{P_{s}}} P_{j}^{-}
\end{aligned}
$$

where

$$
\Delta k_{j}=k_{j}-k_{D}, \quad j=0,1,2
$$

Introducing (32)-(34) into the coupled-mode equations (38)-(39) we obtain for the forward propagating component of the pump

$$
\begin{aligned}
-i \frac{d A_{o}^{+}}{d z}= & \left\{\alpha_{o}\left[1-C \eta\left(\left|A_{o}^{+}\right|^{2}+2\left|A_{o}^{-}\right|^{2}\right)\right]+\Delta k_{o}\right. \\
& \left.+i \alpha_{s c}\right\} A_{o}^{+}+\left\{-\alpha_{o} \xi /\left(1+\xi P_{o}\right)\right. \\
& \left.\cdot A_{o}^{+}\left(A_{o}^{-}\right)^{*}+\kappa\right\} A_{o}^{-}
\end{aligned}
$$

and of the probe and conjugate fields

$$
\begin{aligned}
-i \frac{d A_{j}^{+}}{d z}= & \left\{\alpha _ { o } \left[1-\left(Q_{j}+2 C \eta\right)\left|A_{o}^{+}\right|^{2}-C\left(\xi Q_{j}^{\prime}\right.\right.\right. \\
& \left.\left.+2 \eta)\left|A_{o}^{-}\right|^{2}\right]+\Delta k_{j}+i \alpha_{s c}\right\} A_{j}^{+} \\
& +\left\{-\alpha_{o}\left[Q_{j}+\xi /\left(1+\xi P_{o}\right)+2 C \eta\right]\right. \\
& \left.\cdot\left(A_{o}^{-}\right)^{*} A_{o}^{+}+\kappa\right\} A_{j}^{-} \\
& +\left\{-\alpha_{o}\left(Q_{j}+C \eta\right)\left(A_{o}^{+}\right)^{2}\right\}\left(A_{3-j}^{+}\right)^{*} \\
& +\left\{-\alpha_{o}\left[Q_{j}+C\left(\xi Q_{j}^{\prime}+2 \eta\right)\right] A_{o}^{+} A_{o}^{-}\right\}\left(A_{3-j}^{-}\right)^{*}
\end{aligned}
$$

where

$$
\begin{gathered}
\alpha_{o}=-\frac{\Gamma}{2} a\left(\bar{N}_{b}-N_{o}\right)(\alpha+i) \\
Q_{1}=Q_{2}^{*}=\frac{C}{1+P_{o}-i \Omega \tau_{s}}, \\
Q_{1}^{\prime}=Q_{2}^{\prime *}=\frac{1}{1+\xi\left(P_{o}-i \Omega \tau_{s}\right)} \\
\eta=\epsilon P_{s} \frac{\beta+i}{\alpha+i} \\
\Delta k_{j}=\Delta k_{o}+(-1)^{j-1} \frac{\bar{n} \Omega}{c}=\Delta k_{o}+(-1)^{j-1} \frac{\Omega}{v_{g}} .
\end{gathered}
$$

The backward propagating components are obtained from the corresponding forward components with the substitution $d z \rightarrow-d z$ and ( $+\leftrightarrow-)$ ). In (41)-(46) a linear loss coefficient $\alpha_{s c}$ has been introduced to account for all the linear losses, most due to scattering, of the waveguide. 
Equations (41) and (42) are the equations of FWM in a diffusive gain medium under the linearization approximation. All terms into (42) have a simple physical interpretation. The terms proportional to $Q_{j}$ are due to the mixing into the gain medium of waves with the same $k$ vector: the frequency dependent response typical of a low-pass filter accounts for the non-instantaneous response of the medium, and the dependence on $P_{o}=\left|E_{o}\right|^{2} / P_{s}$ for its saturation. The terms proportional to $Q_{j}^{\prime}$ are due to the mixing of waves with opposite $k$ vectors generating into the gain medium a periodic variation of gain and refractive index, which is smoothed by the carrier diffusion. If we write $Q_{j}^{\prime}$ as

$$
Q_{1}^{\prime}=Q_{2}^{\prime *}=\frac{1}{1+\left|E_{o}\right|^{2} /\left(P_{s} \xi^{-1}\right)-i \Omega \tau_{s} \xi}
$$

one sees right away that these terms have the faster "diffusive" time response $\tau_{D}=\tau_{s} \xi$ and the higher saturation intensity $P_{D}=P_{s} \xi^{-1}$. A fast response and high saturation intensity are as usual together with a weak nonlinearity: these terms are in fact proportional to $\xi$. The time $\tau_{D}$ is related to the lifetime of the carrier grating. This is understood with the following arguments. First, for $D=0$ we have $\xi=1$ and hence $\tau_{D}=\tau_{s}$ : the lifetime of the grating is as expected the same of the lifetime of the excited carriers. Let us consider now the case $\xi \ll 1$, that is, from (24), $4 D \tau_{s} k_{D}^{2} \gg 1$. In this limit, $\tau_{D}=\xi \tau_{s}=1 /\left(4 D k_{D}^{2}\right)$ is the time $t^{*}$ at which the diffusion length $\sqrt{D t^{*}}$ becomes comparable to [to be more precise, equal to $1 /(2 \pi)$ times] the grating period $\pi / k_{D}$. This is the time at which the grating starts to be washed out. The only frequency independent term due to spatial hole burning is the one due to the standing wave generated by the beating of the conterpropagating components of the pump $A_{o}^{+}\left(A_{o}^{-}\right)^{*}$. This term can be regarded as an additional DFB grating induced by the action of the cavity standing wave on the carrier density. This term is also not proportional to $C$ because the beating is generated by waves with the same frequency and hence with the same mode profile.

Finally, we discuss the terms due to the nonlinear gain compression factor. They are the terms proportional to $\eta$ and, through it, to $\epsilon$ are due to the gain and refractive index gratings induced by the gain saturation. They are independent on frequency due to the assumed instantaneous response of the gain saturation on the intensity variations. When we take into account the finite time response of the nonlinear gain saturation, $\epsilon$ and hence $\eta$ is multiplied by

$$
Q_{1}^{\prime \prime}=Q_{2}^{\prime *}=\frac{1}{1+\left|E_{o}\right|^{2} / P_{n l}-i \Omega \tau_{n l}}
$$

in the equations for $A_{1}$ and $A_{2}$, respectively. The terms $P_{n l}$ and $\tau_{n l}$ are the saturation power (much larger than $P_{s}$ ) and the time response of the nonlinear gain. For the interesting case of $P_{n l} \rightarrow \infty$, (47) may be obtained assuming a delayed response of the nonlinearity with delay $\tau_{n l}$, without any hypotesis on the physical mechanism responsible for the nonlinear gain saturation. This may be accomplished by substituting in (9), (29), and (30) the term $\epsilon|E(t)|^{2}$ with

$$
\frac{\epsilon}{\tau_{n l}} \int_{-\infty}^{t} d t^{\prime}\left|E\left(t^{\prime}\right)\right|^{2} \exp -\frac{t-t^{\prime}}{\tau_{n l}} .
$$

With the assumption of a finite time response of the nonlinear gain saturation, (42) acquire a more symmetric form, and can be used also for highly degenerate FWM [23]. The value of the time response of the nonlinear gain depends on its the physical origin, 0.650 ps in InGaAsP if carrier heating [24], of the order of $0.3 \mathrm{ps}$ if spectral hole burning.

Now, we connect the laser internal fields to the outside radiation. This is done by writing the boundary conditions

$$
\begin{gathered}
E_{j, \text { out }}(L)=\sqrt{1-R_{2}} E_{j}^{+}(L)-r_{2}^{*} E_{j, \text { in }}(L) \\
E_{j}^{-}(L)=r_{2} E_{j}^{+}(L)-\sqrt{1-R_{2}} E_{j, \text { in }}(L) \\
E_{j}^{+}(0)=r_{1} E_{j}^{-}(0)+\sqrt{1-R_{1}} E_{j, \text { in }}(0) \\
E_{j, \text { out }}(0)=\sqrt{1-R_{1}} E_{j}^{-}(0)+r_{1}^{*} E_{j, \text { in }}(0)
\end{gathered}
$$

where

$$
\begin{aligned}
r_{h} & =\sqrt{R_{j}} e^{i \phi_{h}} \\
E_{j}^{+}(z) & =A_{j}^{+}(z) \sqrt{P_{s} A_{\text {eff }}}
\end{aligned}
$$

and $R_{h}$ and $\phi_{h}$ are the power reflectivity and the phase of the facet $h$. The square modulus of the input and output waves $E_{j, \text { out }}$ and $E_{j, \text { in }}$ is normalized to give the output and input power at the left- and right-end sides of the waveguide.

\section{Mean Field Approximation}

In general, in a laser above threshold the set of equations (41)-(42) with the boundary conditions (48)-(53) calls for a numerical solution. Yet in the limit of $R_{1}=R_{2}$ $=R \rightarrow 1$ when the threshold gain of the laser tends to zero, one can take advantage of the homogeneity of all the interacting fields to find analytical solutions. In this limit, where the mirror losses are negligible with respect to the scattering losses, one has

$$
\begin{aligned}
\boldsymbol{A}_{j}^{+}(z) & =A_{j}^{-}(z)=\text { independent on } z \\
& =\frac{A_{j}}{\sqrt{2}} \quad j=0,1,2
\end{aligned}
$$

so that from the equation for the pump (41), by making use of (54) for $j=0$ and of $A_{o} \neq 0$, we get

$$
\begin{gathered}
\alpha_{o}\left[1-\left(\frac{3}{2} C \eta+\frac{1}{2} \frac{\xi}{1+\xi P_{o}}\right)\left|A_{o}\right|^{2}\right] . \\
+\Delta k_{o}+i \alpha_{s c}+\kappa=0
\end{gathered}
$$


By making use of (54) and (55) the equations for the probe and conjugate fields (42) becomes

$$
\begin{aligned}
\left\{-\alpha_{o}\right. & {\left.\left[Q_{j}+\frac{C}{2}\left(3 \eta+\xi Q_{j}^{\prime}\right)\right]\left|A_{o}\right|^{2}+(-1)^{j-1} \frac{\Omega}{v_{g}}\right\} \times A_{j} } \\
& +\left\{-\alpha_{o}\left[Q_{j}+\frac{C}{2}\left(3 \eta+\xi Q_{j}^{\prime}\right)\right] A_{o}^{2}\right\} \times A_{3-j}^{*} \\
= & -\sqrt{2} F_{j}
\end{aligned}
$$

where the $Q_{j}$ 's are given by (44) with $P_{o}=\left|A_{o}\right|^{2}$. In (56) the source term

$$
F_{j}=\left\{\begin{array}{cc}
\frac{A_{\text {in }}}{2 L} & j=1 \\
0 & j=2
\end{array}\right.
$$

has been introduced to account for the injection of the only probe field from the outside. In (57) $L$ is the laser length and the field $A_{\text {in }}$ is related to the field $E_{1, \text { in }}$ impinging upon the external facet of the laser by the relation

$$
A_{\text {in }}=\sqrt{\eta_{\text {eff }}(1-R)} \frac{E_{1, \text { in }}}{\sqrt{P_{s} \bar{A}_{\text {eff }}}}
$$

where $\eta_{\text {eff }}$ is a coupling efficiency. The solution of the set of equations (56) is

$$
\begin{aligned}
& A_{\mathrm{l}}(\Omega)=\frac{H_{1}(\Omega)}{H_{o}(\Omega)} \frac{A_{\text {in }}}{\sqrt{2} L} \\
& A_{2}^{*}(\Omega)=\frac{H_{2}(\Omega)}{H_{o}(\Omega)} \frac{A_{\text {in }}}{\sqrt{2} L}
\end{aligned}
$$

where

$$
\begin{aligned}
H_{o}(\Omega)= & \frac{\Omega}{v_{g}}\left\{-\frac{\Omega}{v_{g}}+\alpha_{o}\left[Q_{1}+\frac{C}{2}\left(3 \eta+\xi Q_{1}^{\prime}\right)\right]\left|A_{o}\right|^{2}\right. \\
& \left.-\alpha_{o}^{*}\left[Q_{1}+\frac{Q}{2}\left(3 \eta^{*}+\xi Q_{1}^{\prime}\right]\right]\left|A_{o}\right|^{2}\right\} \\
H_{1}(\Omega)= & -\alpha_{o}^{*}\left[Q_{1}+\frac{C}{2}\left(3 \eta^{*}+\xi Q_{1}^{\prime}\right)\right]\left|A_{o}\right|^{2}-\frac{\Omega}{v_{g}} \\
H_{2}(\Omega)= & \alpha_{o}^{*}\left[Q_{1}+\frac{C}{2}\left(3 \eta^{*}+\xi Q_{1}^{\prime}\right]\right]\left(A_{o}^{*}\right)^{2}
\end{aligned}
$$

The output power per facet is given by

$$
\left|E_{j, \text { out }}\right|^{2}=\frac{1}{2}(1-R) P_{s} A_{\text {eff }}\left|A_{j}\right|^{2} .
$$

If we take into account the finite time response of the gain compression term, the term $\eta^{*}$ in the previous equation is multiplied for $Q_{1}^{\prime \prime}$ given by (47) with $P_{o}=\left|A_{o}\right|^{2}$.
The intensity of the probe and conjugate fields diverges for $\Omega=0$. This divergence is unphysical because when the frequency of the probe approaches the pump frequency the laser starts running in injection locking. This regime is not described by our model which assumes that the field of the laser free running is not affected, to lowest order, by the injected radiation.

Equations (56) and (64) have been obtained for lasers FP in the limit of high facet reflectivities. We have checked by direct integration of equation (41) and (42) that this approximation is a good one even for mirror reflectivities $R$ of the order of 0.32 , if to the power $\left|A_{o}\right|^{2}$ is given the meaning of the average intracavity power. For DFB lasers, the shape of FWM efficiency obtained from (56) and (64) is still in very good agreement with the numerical analysis, even if the values of the calculated FWM response differ from those obtained numerically by a factor, which is anyway independent on frequency.

It is worth to notice that a result similar to (56) can also be obtained by assuming from the beginning the carrier density and the fields as homogeneous. The equation for the carrier density is still, in this approach, given by (8), without the diffusion term, and the equation describing the field-matter interaction is the usual rate equation for the carrier density which is familiar from the theory of the injection locking

$$
\begin{aligned}
\frac{d E(t)}{d t}= & -\frac{1}{2}\left\{\gamma-a\left(N-N_{o}\right)\left[\left(1-\epsilon|E|^{2}\right)\right.\right. \\
& \left.\left.+i\left(\alpha-\beta \epsilon|E|^{2}\right)\right]\right\} E-\kappa_{c} E_{1, \text { in }} \exp (-i \Omega t)
\end{aligned}
$$

In this equation, $\kappa_{c}$ is a coupling constant which relates the external input probe field with the intracavity field and $\gamma$ are the cavity losses. Expanding the internal field and the carrier density as in (2) and (10) respectively, and equating the coefficients of equal time harmonic terms, we obtain a result which, though similar to the already obtained result, yet has some differences with (56). First of all, the terms proportional to $\xi$ due to the spatial hole burning induced by the counterpropagating waves are not present in the result of this simplified approach, and cannot be obtained by any model which neglects from the beginning the spatial inhomogeneity of the laser. Then, the factor $3 / 2$ in front of $\eta$ is substituted with 1 in the result of the rate equation model. This difference arises from the fact that the "inhomogeneous" approach takes into account the presence of the grating of nonlinear gain saturation which is generated by the cavity standing wave, which is instead completely neglected by the simplified approach.

In the particular case of $\eta=\xi=0,(59)-(63)$ acquire the more transparent form

$$
A_{1}(\Omega)=\frac{-\frac{1}{2} g \Gamma(1+i \alpha) C\left|A_{o}\right|^{2}+i \frac{\Omega}{v_{g}}\left(1+\left|A_{o}\right|^{2}-i \Omega \tau_{s}\right)}{\frac{\Omega}{v_{g}}\left[\left(1+\left|A_{o}\right|^{2}\right) i \frac{\Omega}{v_{g}}+\frac{\tau_{s}}{v_{g}}\left(\Omega^{2}-\Omega_{R}^{2}\right)\right]} \frac{A_{\mathrm{in}}}{\sqrt{2} L} .
$$




$$
A_{2}^{*}(\Omega)=\frac{\frac{1}{2} g \Gamma(1+i \alpha) C\left(A_{o}^{*}\right)^{2}}{\frac{\Omega}{v_{g}}\left[\left(1+\left|A_{o}\right|^{2}\right) i \frac{\Omega}{v_{g}}+\frac{\tau_{s}}{v_{g}}\left(\Omega^{2}-\Omega_{R}^{2}\right)\right]} \frac{A_{\text {in }}}{\sqrt{2} L}
$$

where

$$
g=a\left(\bar{N}_{b}-N_{o}\right)
$$

is the laser gain, sum of the threshold gain and of the scattering losses, and

$$
\Omega_{R}=\left(g \Gamma C\left|A_{o}\right|^{2} \frac{v_{g}}{\tau_{s}}\right)^{1 / 2}
$$

is the laser resonance frequency. A resonant enhancement of the FWM efficiency at the frequency $\Omega_{R}$, which is directly proportional to the square root of the optical intensity of the laser, is predicted by this equations. They also show that the intensities of FWM signals are proportional to the factor $\left(1+\alpha^{2}\right)$.

Gain compression and/or carrier density grating smooth the resonance peaks of the FWM spectrum. The reason for this is clear, if one thinks about the origin of the relaxation oscillations. When a fluctuation of the photon density is generated, this affect the carrier density, and hence the gain, with a delay due to the lifetime of the excited carriers. This generates oscillations of the carrier and photon densities, which are consequently smoothed when a fast dependence of the gain (nonlinear gain) or losses (carrier grating) on the field is introduced.

Before discussing the result of the theory, and how it matches with the experimental results, we will address in the next section the effect on FWM of the finite linewidth of both pump and signal. The results of this section have been already published elsewhere [25], together with their experimental check. We will repeat here the very simple theoretical analysis since the broadening of the conjugate line has important consequences on the interpretation of the experimental data of the FWM response, as we will see later on.

\section{EFFECT of NoISE}

Equations (66) and (67) assume that both the pump and the probe are free of any noise disturbance. This is in general not the case because both pump and signal are affected by amplitude and phase noise, the latter being responsible for the finite linewidth of the field autoconvolution spectrum. We will assume in the following that the amplitude noise is negligible with respect to phase noise, a usual approximation when dealing with linewidths. The effect of phase noise of pump and signal can be easily accounted for in our model, by substituting $A_{o}$ and $A_{\text {in }}$ with $A_{o} e^{i \phi_{o}(t)}$ and $A_{\text {in }} e^{i \phi_{\text {in }}(t)}$, respectively, where $\phi_{o}(t)$ and $\phi_{\text {in }}(t)$ are the pump and signal phase noise. This substitution is not quite rigorous, because equations (66) and (67) are already in frequency domain, but is a good approximation when, as usual, the separation between pump, signal and conjugate is much larger than the line-

width of the fields. This means that we consider the effect on FWM of the low frequency content of the phase noise only, the same that gives rise to the linewidth of the fields. This component is much slower than the inverse of the beating frequency $\Omega$ and consequently we can assume that the output fields $A_{1}(\Omega)$ and $A_{2}(\Omega)$ adiabatically follow the phase noise of signal and pump.

To simplify the notations, let us write $(65)$ and $(66)$ in the formal way:

$$
\begin{aligned}
& A_{1}(\Omega)=F_{1}\left(\Omega,\left|A_{o}\right|^{2}\right) A_{\text {in }} \\
& A_{2}^{*}(\Omega)=F_{2}\left(\Omega,\left|A_{o}\right|^{2}\right)\left(A_{o}^{*}\right)^{2} A_{\text {in }}
\end{aligned}
$$

Introducing the phase noise of pump and signal they become

$$
\begin{aligned}
& A_{1}(\Omega, t)=F_{1}\left(\Omega,\left|A_{o}\right|^{2}\right) A_{\text {in }} e^{i \phi_{\text {in }}(t)} \\
& A_{2}^{*}(\Omega, t)=F_{2}\left(\Omega,\left|A_{o}\right|^{2}\right)\left(A_{o}^{*}\right)^{2} e^{-2 i \phi_{o}(t)} A_{\text {in }} e^{i \phi_{\text {in }}(t)} .
\end{aligned}
$$

The field autoconvolutions write

$$
\begin{aligned}
\left\langle A_{1}(\Omega, t+T) A_{1}^{*}(\Omega, t)\right\rangle= & \left|F_{1}\left(\Omega,\left|A_{o}\right|^{2}\right)\right|^{2}\left|A_{\text {in }}\right|^{2} \\
& \cdot\left\langle e^{i\left[\phi_{\text {in }}(t+T)-\phi_{\text {in }}(t)\right]}\right\rangle \\
\left\langle A_{2}^{*}(\Omega, t+T) A_{2}(\Omega, t)\right\rangle= & \left|F_{2}\left(\Omega,\left|A_{o}\right|^{2}\right)\right|^{2}\left|A_{o}\right|^{4}\left|A_{\text {in }}\right|^{2} \\
& \times\left\langle e^{-2 i\left[\phi_{o}(t+T)-\phi_{o}(t)\right]}\right. \\
& \left.\times e^{i\left[\phi_{\text {in }}(t+T)-\phi_{\text {in }}(t)\right]}\right\rangle .
\end{aligned}
$$

It is well known that the phase diffusion for low frequencies is a random walk of variance

$$
\left\langle\left[\phi_{j}(t+T)-\phi_{j}(t)\right]^{2}\right\rangle=D_{j}|T|
$$

where the diffusion constant $D_{j}$ is related to the linewidth by the relationship [26]

$$
\Delta \nu_{j}=D_{j} /(4 \pi)
$$

By using the property of the Gaussian processes

$$
\left\langle e^{i \lambda\left[\phi_{j}(t+T)-\phi_{j}(t)\right]}\right\rangle=e^{-\lambda^{2} D_{j}|T| / 2}
$$

and from the independence of the phase noise of pump and signal we get

$$
\begin{aligned}
\left\langle A_{1}(\Omega, t+T) A_{i}^{*}(\Omega, t)\right\rangle= & \left|F_{1}\left(\Omega,\left|A_{o}\right|^{2}\right)\right|^{2} \\
& \cdot\left|A_{\text {in }}\right|^{2} e^{-D_{\text {in }}|T| / 2} \\
\left\langle A_{2}^{*}(\Omega, t+T) A_{2}(\Omega, t)\right\rangle= & \left|F_{2}\left(\Omega,\left|A_{o}\right|^{2}\right)\right|^{2}\left|A_{o}\right|^{4} \\
& \cdot\left|A_{\text {in }}\right|^{2} e^{-4 D_{o}|T| / 2} e^{-D_{\text {in }}|T| / 2} .
\end{aligned}
$$


Fourier transforming with respect to the slow time scale $T$ we get the power spectrum of $A_{1}$ and $A_{2}$ :

$$
\left\langle\left|A_{1}(\Omega, \omega)\right|^{2}\right\rangle=\left|F_{1}\left(\Omega,\left|A_{o}\right|^{2}\right)\right|^{2}\left|A_{\text {in }}\right|^{2} \frac{4 \pi \Delta \nu_{1}}{\omega^{2}+\left(2 \pi \Delta \nu_{1}\right)^{2}}
$$

$$
\begin{aligned}
\left\langle\left|A_{2}(\Omega, \omega)\right|^{2}\right\rangle= & \left|F_{2}\left(\Omega,\left|A_{o}\right|^{2}\right)\right|^{2}\left|A_{o}\right|^{4}\left|A_{\text {in }}\right|^{2} \\
& \cdot \frac{4 \pi \Delta \nu_{2}}{\omega^{2}+\left(2 \pi \Delta \nu_{2}\right)^{2}}
\end{aligned}
$$

where

$$
\begin{aligned}
& \Delta \nu_{1}=\Delta \nu_{\text {in }} \\
& \Delta \nu_{2}=4 \Delta \nu_{o}+\Delta \nu_{\text {in }}
\end{aligned}
$$

and $\omega$ is the angular frequency deviation from the center frequency of the probe and conjugate.

The linewidth of the probe output is the same of the input. The linewidth of the conjugate is much broader of those of pump and probe, and is very sensitive on the pump linewidth. The integral over all the "slow" frequencies is however independent on the linewidths, and is the same as the ideal case of absence of phase noise. Yet, the peak value of probe and conjugate in frequency domain is largerly affected by phase noise. In the special case of equal linewidths of pump and probe, the peak of the conjugate is five times lower than that of the probe, assuming that their total energies are the same.

In an experiment, the broadening of the spectrum of the conjugate may lead to lose part of the energy contained in the wings of the spectrum if an interferometer is used in the measurement. This seems to be consistent with our experimental results. By simply inspecting Fig. 2(b), one realizes that the experimental points which refer to the conjugate can be practically superimposed to the corresponding ones relative to the probe by a constant shift in logarithmic scale. This implies a constant factor, in this case 1.5-2, between the probe and conjugate output power. This is exactly the expected behavior if a constant fraction of the conjugate power is lost in the measurement (note that pump and probe have roughly the same linewidth in all the experimental points). When the pump laser is biased at higher currents its linewidth narrows, and the difference between probe and conjugate practically disappears [see Fig. 2(a)], as expected from (81)-(84).

To give a quantitative evaluation of the effect of the noise in the experiment, we assume that the transmission of the interferometer is a Lorenzian centered at the peak of the probe or conjugate of width $\Delta \nu_{m}$. Multiplying (81) and (82) for the interferometer transmission and integrating over all the frequencies, we obtain

$$
\left\langle\left|A_{1}(\Omega)\right|^{2}\right\rangle=\left|F_{1}\left(\Omega,\left|A_{o}\right|^{2}\right)\right|^{2}\left|A_{\text {in }}\right|^{2} \frac{\Delta \nu_{m}}{\Delta \nu_{m}+\Delta \nu_{1}}
$$

$$
\begin{aligned}
\left\langle\left|A_{2}(\Omega, \omega)\right|^{2}\right\rangle & =\left|F_{2}\left(\Omega,\left|A_{o}\right|^{2}\right)\right|^{2}\left|A_{o}\right|^{4}\left|A_{\text {in }}\right|^{2} \\
& \cdot \frac{\Delta \nu_{m}}{\Delta \nu_{m}+\Delta \nu_{2}} .
\end{aligned}
$$

In the experiment, $\Delta \nu_{m}$ was around $600 \mathrm{MHz}$. If we assume $\Delta \nu_{\text {in }}=\Delta \nu_{o}=40 \mathrm{MHz}$, for the set of measurements with the lower injection current, we get that the measured conjugate intensity is only the $75 \%$ of the total, while the measured probe intensity is the $94 \%$ of the total. We will use in the next section (85) and (86) to compare with the experimental results.

\section{Results}

In Figs. 3-5 we show the experimental results together with the theoretical FWM response. The value of the parameters for the theoretical plots are listed in Table I. When no damping mechanisms are introduced, an evident enhancement of the FWM response close to the resonance peaks shows up. In Fig. 4 only the gain saturation is introduced and the resonant enhancement damps out. In Fig. 5 no gain saturation is assumed $(\eta=0)$, but the effect of the induced carrier grating is considered by assuming $\xi$ $=6 \times 10^{-2}$. This is equivalent to a value of the diffusion constant $D$ of $1 \mathrm{~cm}^{2} / \mathrm{s}$. The relaxation oscillations are still damped, yet they are damped less compared to the case in which gain saturation is introduced. If we increase $\xi$ by assuming a lower value for the diffusion constant, the relaxation resonance peaks does not reduce significantly, at least for reasonable values for $D$. By increasing $\xi$, in fact, the lifetime of the grating $\tau_{D}=\xi \tau_{s}$ also increases approaching $\tau_{s}$. The efficiency of the grating to damp out the relaxation oscillations consequently reduces since, as already mentioned, this efficiency is related to the presence of gain, or loss, mechanisms much faster than the carrier dynamics.

From the above results, it seems that our set of measurements is better described by assuming that the gain saturation rather than the carrier grating damps the resonant enhancement of the FWM efficiency at the relaxation oscillation frequency.

\section{Discussion}

It is worth to compare our results of FWM in DFB semiconductor lasers with the results already published of FWM in amplifiers and FP lasers. Our data show a high degree of symmetry of the probe amplification for positive and negative pump-probe detunings. Although we are not aware of published results showing the probe amplification for both sides of the pump-probe detunings in FP lasers ([4] and [5] presents only one side of the spectrum, while [6] does not report any investigation of the FWM response versus frequency), our theory predicts this behavior for FWM of lasers above threshold no matter if they are FP or DFB.

Our experimental data show that probe and conjugate outputs have almost the same power level over the whole range of measured frequencies. The experimental data of 


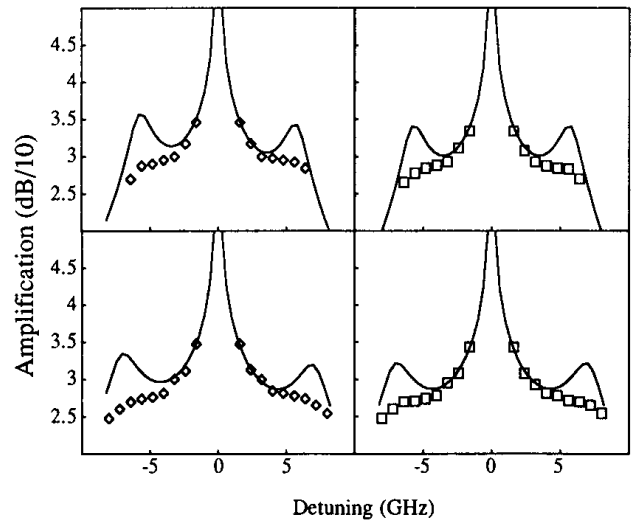

Fig. 3. Experimental results (diamonds for probe and squares for conjugate waves) together with the theoretical FWM response (solid line) when no mechanisms to damp the FWM response are present. In the upper part of the figure the test laser is biased at $50 \mathrm{~mA}$, in the lower part at $70 \mathrm{~mA}$.

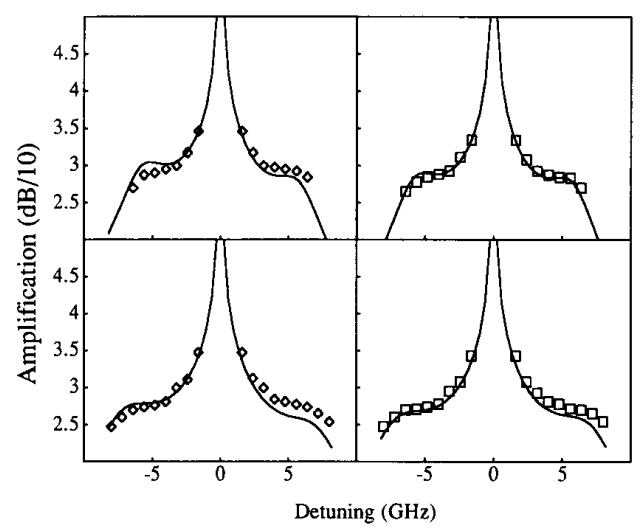

Fig. 4. Experimental results (diamonds for probe and squares for conjugate waves) together with the theoretical FWM response (solid line) when only the gain saturation is taken into account. In the upper part of the figure the test laser is biased at $50 \mathrm{~mA}$, in the lower part at $70 \mathrm{~mA}$.

FWM in FP lasers reported in [4] and [5] show at very low frequency detunings (less than $1 \mathrm{GHz}$ ) the output power of the conjugate much lower than the output power of the probe. We do not have any data for frequency detunings that low. Yet, such a difference of power levels is not predicted by our theory. A possible cause for the discrepancy is the perturbative approach we followed in our theory, which fails when the probe and conjugate powers becomes comparable to the pump power. Another reason might be the broadening of the conjugate line that lowers the detected conjugate power if the resolution of the Fabry-Perot used to resolve the fields is too high. A high resolution is necessary when the conjugate line is very close to the pump line, that is for very low values of the detunings. The data for very low detunings reported in literature [4], [5] might be consequently affected by large errors. Both our data and the data reported in literature for FP lasers [4], [5] show at higher detunings (larger than $1 \mathrm{GHz}$ ) probe and conjugate outputs almost equal.

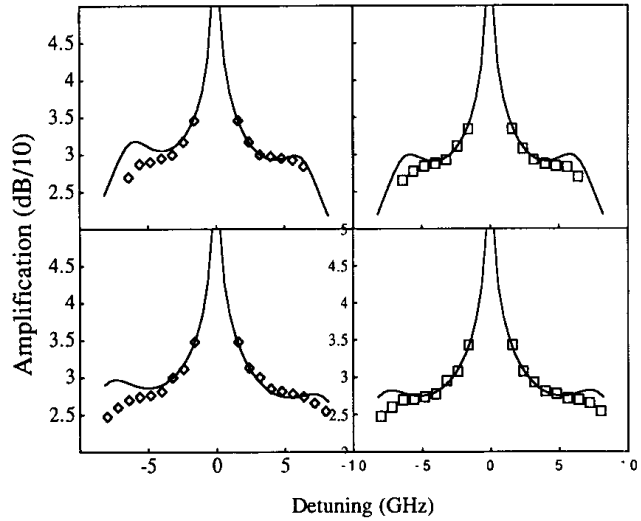

Fig. 5. Experimental results (diamonds for probe and squares for conjugate waves) together with the theoretical FWM response (solid line) when the effect of the diffusion is considered. In the upper part of the figure the test laser is biased at $50 \mathrm{~mA}$, in the lower part at $70 \mathrm{~mA}$.

TABLE I

\begin{tabular}{lccc}
\hline & Fig. 3 & Fig. 4 & Fig. 5 \\
\hline$C$ & 1 & 1 & 1 \\
$P_{o}$ & $1.8 / 2.7$ & $1.8 / 2.7$ & $1.8 / 2.7$ \\
$\tau_{s}(\mathrm{~s})$ & $0.3 \cdot 10^{-9}$ & $0.3 \cdot 10^{-9}$ & $0.3 \cdot 10^{-9}$ \\
$v_{g}(\mathrm{~m} / \mathrm{s})$ & $0.75 \cdot 10^{8}$ & $0.75 \cdot 10^{8}$ & $0.75 \cdot 10^{8}$ \\
$\alpha$ & 6 & 6 & 6 \\
$g\left(\mathrm{~cm}^{-1}\right)$ & 60 & 60 & 60 \\
$L(\mathrm{~m})$ & $300 \cdot 10^{-6}$ & $300 \cdot 10^{-6}$ & $300 \cdot 10^{-6}$ \\
$\xi$ & 0 & 0 & $6 \cdot 10^{-2}$ \\
$\beta$ & 0 & 0 & 0 \\
$\epsilon$ & 0 & $1.5 \cdot 10^{-2}$ & 0 \\
\hline
\end{tabular}

In general, we do not see any striking difference between the experimental results of FWM in FP and DFB lasers. Traveling wave equations (41) and (42) take into account the index grating. Yet the index grating has no effect in the final solutions (59)-(64) since those solutions are obtained with the homogeneous field approximation. We do not believe, however, that the effect of the grating is very important. It only provides the phase matching conditions for counterpropagating waves at the same frequency; it affects the FWM response by only changing the field distributions of the interacting fields, beside giving rise to the cavity effect which is, however, present also in FP lasers. Obviously, a static grating cannot contribute to the energy exchanges between waves at different frequencies, as dynamic gratings do.

\section{CONCLUSION}

Experimental and theoretical analysis on nearly degenerate four-wave mixing in a distributed feedback semiconductor laser working above threshold have been reported. Measurements at two different values of bias of the test laser show that the lineshape of both the probe and the conjugate waves are symmetric with respect to the zero pump-probe detuning. The effective FWM bandwidth is mainly determined by relaxation oscillation fre- 
quency of the laser and it increases when the bias is increased. A theoretical analysis has shown that the model of population pulsation of [6] is adequate also for FWM of semiconductor lasers above threshold, when the effects of the nonlinear gain compression and of the spatial hole burning are properly taken into account. The effect of the modulation of the carrier density and the spatial hole burning cannot explain alone the measured FWM spectra close to the laser relaxation frequency. Good agreement between theory and experiment has been obtained when a nonlinear gain compression term is introduced in the model and the effect of the phase noise of the laser is taken into account.

\section{ACKNOWLEDGMENT}

We acknowledge fruitful discussions with $P$. Spano; without his continuous encouragements this work would have never been done.

\section{REFERENCES}

[1] R. A. Fisher, Optical Phase Conjugation. New York: Academic, 1983.

[2] Y. R. Shen, The Principle of Nonlinear Optics. New York: Wiley, 1984.

[3] H. Nakajima and R. Frey, "Observation of bistable reflectivity of a phase conjugated signal through intracavity nearly degenerate fourwave mixing," Phys. Rev. Lett., vol. 54, pp. 1798-1801, 1985.

[4] _ " "Intracavity nearly degenerate four-wave mixing in a (GaAl)As semiconductor laser," Appl. Phys. Lett., vol. 47, pp. 769-771, 1985.

[5] _ , "Collinear nearly degenerate four-wave mixing in intracavity amplifying media," IEEE J. Quantum Electron., vol. QE-22, pp. $1349-1354,1986$.

[6] R. Nietzke, P. Fenz, W. Elsässer, and E. O. Göbel, "Cascade fourwave mixing in semiconductor lasers," Appl. Phys. Lett., vol. 51 , pp. $1298-1300,1987$

[7] A. P. Bogatov, P. G. Eliseev, and B. N. Sverdlov, "Anomalous interation of spectral modes in a semiconductor laser," IEEE J. Quantum Electron., vol. QE-11, pp. 510-515, 1975.

[8] G. P. Agrawal, "Population pulsations and nondegerate four-wave mixing in semiconductor lasers and amplifiers," J. Opt. Soc. Amer. $B$, vol. 5, pp. $147-158,1988$.

[9] K. Inoue, T. Mukai, and T. Saitoh, "Nearly degenerate four-wave mixing in a traveling-wave semiconductor laser amplifier," Appl. Phys. Lett., vol. 51, pp. 1051-1053, 1987.

[10] F. Favre and D. Le Guen, "Four-wave mixing in traveling-wave semiconductor laser amplifiers," IEEE J. Quantum Electron., vol. 26 , pp. 858-864, 1990

[11] T. Mukai and T. Saitoh, "Detuning characteristics and conversion efficiency of nearly degenerate four-wave mixing in a $1.5 \mathrm{~mm}$ traveling-wave semiconductor laser amplifier," IEEE J. Quantum Electron., vol. 26, pp. 865-875, 1990.

[12] G. P. Agrawal and N. K. Dutta, Long Wavelength Semiconductor Lasers. New York: Van Nostrand Reinhold, 1984

[13] T. Mukai and Y. Yamamoto, "Gain, frequency bandwidth, and saturation output power of AIGaAs DH laser amplifiers," IEEE J. Quantum Electron., vol. QE-17, pp. 1028-1034, 1981.

[14] R. Hui, A. Mecozzi, A. D'Ottavi, and P. Spano, "Novel measurement technique of a factor in DFB semiconductor lasers by injection locking," Electron. Lett., vol. 26, pp. 997-998, 1990.

[15] I. Petitbon, P. Gallion, G. Debarge, and C. Chabran, "Locking bandwidth and relaxation oscillation of an injection-locked semiconductor laser," IEEE J. Quantum Electron., vol. 24, pp. 148-154, 1988

[16] D. J. Chanuin, "Effect of gain saturation on injection laser switching," J. Appl. Phys., vol. 50, pp. 3858-3860, 1979.
[17] C. B. Su and V. A. Lanzisera, "Ultra-high-speed modulation of 1.3 $\mathrm{mm}$ InGaAsP diode laser," IEEE J. Quantum Electron., vol. QE-22, pp. $1568-1578,1986$.

[18] M. Asada and Y. Suematsu, "Density-matrix theory of semiconductor lasers with relaxation Broadening model-gain and gain-suppression in semiconductor lasers," IEEE J. Quantum Electron., vol. QE21, pp. 434-442, 1985.

[19] G. P. Agrawal, "Gain nonlinearities in semiconductor lasers: Theory and application to distributed feedback laser," IEEE J. Quantum Electron., vol. QE-23, pp. 860-868, 1985.

[20] M. P. Kesler and E. P. Ippen, "Subpicopsecond gain dynamics in GaAlAs laser diodes," Appl. Phys. Lett., vol. 51, pp. 1765-1767, 1987.

[21] R. Fraukenberger and R. Schimpe, "Measurement of the gain saturation spectrum in InGaAsP diode lasers," Appl. Phys. Lett., vol. 57. pp. $2520-252,1990$

[22] C. B. Su, "Dielectric grating induced by cavity standing wave as a new explanation of the the origin of nonlinear gain in semiconductor diode lasers," Electron. Lett., vol. 24, pp. 370-371, 1988.

[23] L. F. Tiemeijer, "Effects of nonlinear gain on four-wave mixing and asymetric gain saturation in a semiconductor laser amplifier," Appl. Phys. Lett., vol. 59, pp. 499-501, 1991.

[24] K. L. Hall, J. Mark, E. P. Ippen, and G. Eisenstein, "Femtosecond gain dynamics in InGaAsP optical amplifier," Appl. Phys. Lett., vol. 56, pp. $1740-1742,1990$.

[25] R. Hui and A. Mecozzi, "Phase noise of four wave mixing of semiconductor lasers," Appl. Phys. Lett., vol. 60, pp. 2454-2456, 1992.

[26] Y. Yamamoto and H. A. Haus, "Commutation relations and laser linewidth," Phys. Rev. A, vol. 41, pp. 5164-5170, 1990.

Antonio Mecozzi, for a photograph and biography, see p. 996 of the March 1993 issue of this JourNaL.

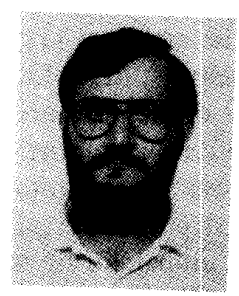

Alessandro D'Ottavi was born in Rome, Italy, on January 29,1962 . He received the physics degree in 1988 from the University of Rome.

$\mathrm{He}$ is currently working in the field of semiconductor lasers at the Fondazione Ugo Bordoni.

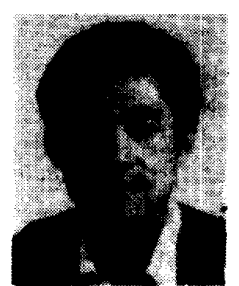

Rongqing Hui was born in Anhui, China, on June 30,1957 . He received the B.Sc. degree in microwave communication and the M.Sc. degree in lightwave technology, both from Beijing University of Posts and Telecommunications, Beijing. China, in 1982 and 1987, respectively.

From 1982 to 1985 he taught at the Department of Physics, Anhui University, where his research interest was in optical fiber sensors. From 1985 to 1989 he worked in the Department of Electronics Engineering, Beijing University of Posts and Telecommunications, where he conducted research into coherent optical transmission systems, single-mode single-polarized optical fibers, and semiconductor lasers with optical feedback. During 1989 and 1990 he held a research fellowship at the Fondazione Ugo Bordoni, Rome, Italy, where his research interests were optical bistability, four-wave mixing, and optical injection locking of semiconductor lasers. $\mathrm{He}$ is now with the Electronics Department, Politecnico di Torino, Torino, Italy. He also holds a research fellowship from the Italian Telecommunication Research Center (CSELT), Torino, Italy. His main research interests are semiconductor laser devices and coherent optical transmission systems. 